\title{
Analysis and Testing of Chain Characteristics and Rheological Properties for Magnetorheological Fluid
}

\author{
Song Chen, ${ }^{1,2}$ Jin Huang, ${ }^{2}$ Hongyu Shu, ${ }^{1}$ Tiger Sun, ${ }^{1}$ and Kailin Jian' \\ ${ }^{1}$ College of Resources and Environmental, Chongqing University, Chongqing 400044, China \\ ${ }^{2}$ College of Mechanical Engineering, Chongqing University of Technology, Chongqing 400054, China \\ Correspondence should be addressed to Jin Huang; jhuangcq@sohu.com
}

Received 16 July 2013; Accepted 9 October 2013

Academic Editor: Xing Chen

Copyright (c) 2013 Song Chen et al. This is an open access article distributed under the Creative Commons Attribution License, which permits unrestricted use, distribution, and reproduction in any medium, provided the original work is properly cited.

Digital holographic microscopy is presented in this study, which can measure the magnetorheological (MR) fluid in different volume fractions of particles and different magnetic field strengths. Based on the chain structure of magnetic particle under applied magnetic field, the relationships between shear yield stress, magnetic field, size, and volume fraction of MR fluid in two parallel discs are established. In this experiment, we choose three MR fluid samples to check the rheological properties of MR fluid and to obtain the material parameters with the test equipment of MR fluid; the conclusion is effective.

\section{Introduction}

Magnetorheological (MR) fluids are suspensions of micronsized, magnetizable particles in a carrier fluid such as synthetic oil and silicone oil, which are regarded as the intelligent materials that respond to an applied magnetic field with a change in their rheological properties. In the absence of an applied magnetic field, MR fluids exhibit Newtonian fluidlike behavior. Upon application of a magnetic field, the polarization between two induced dipoles causes the suspended particles in the MR fluids to form a chain-like microstructure aligned with the direction of applied magnetic field. The magnetic chain structure changes the rheological properties of the suspension. Altering the strength of the applied magnetic field precisely and proportionally controls the shear yield strength of the fluids $[1,2]$. Based on the mechanical characteristics, the fluids can be used in the controllable energy-dissipating applications such as dampers [3, 4], valves $[5,6]$, and clutches and brakes $[7,8]$.

Experiments showed that many other factors affect the macroscopic properties of an MR fluid. It mainly includes the applied magnetic field strength, the size and gradation, and the property and volume fraction of the particles; the property of the carrier fluid and the additives. Noma et al. [9] found that Fe nanoparticles synthesized by the arc plasma method exhibited a high saturation magnetization and may be useful for MR fluids. Ekwebelam and See [10] explored the yielding behavior and enhanced stress response exhibited by bidisperse MR fluid over monodisperse systems. He found that the stress enhancement in bidisperse suspensions is likely to be due to the population and orientation of interacting large particles in the bidisperse suspensions. Jang et al. [11] studied the behavioral model for magnetorheological fluid under a magnetic field using Lekner summation method. Pacull et al. [12] studied the effect of polar interactions on the magnetorheology of silica-coated magnetite suspensions in oil media reported. He suggested that the nonnegligible interfacial interactions are responsible for both the absence of MR effect in hydrophobic samples and the low yield stress in hydrophilic suspensions. To resolve the sedimentation of carbonyl iron (CI) based MR fluid, Fang et al. [13] introduced fibrous single-walled carbon nanotube (SWNT) into carbonyl iron (CI) suspension as additives.

\section{Chain Characteristics of MR Fluid}

2.1. Chain Process. When a magnetic field is applied, the magnetic particles in MR fluid are moving orderly that causes the suspended particles to attract each other under the action 


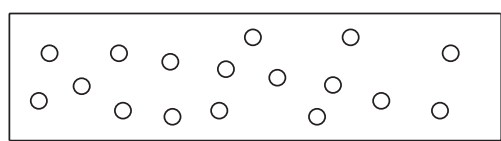

(a)

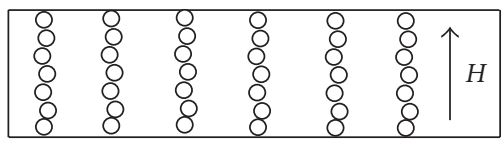

(c)

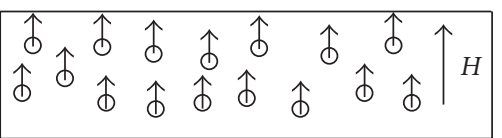

(b)

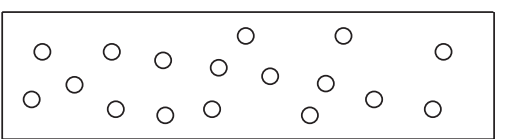

(d)

FIGURE 1: The chain process of MR fluid.

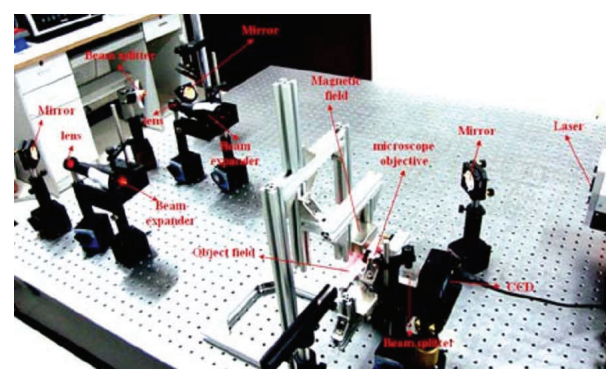

FIGURE 2: The experimental device of measurement of MR fluids by digital microholography.

of magnetic force to form a chain-like microstructure along the field direction; meanwhile the chain process of MR fluid occurrs, as shown in Figure 1. The distribution of particles in MR fluid without the magnetic field is shown in Figure 1(a), the dynamic yield stress is zero in this case. The chain structure of particles in MR fluid under the magnetic field is shown in Figure 1(b). Figure 1(c) shows that the number and diameter of chain will increase with the applied magnetic field and the dynamic yield stress and apparent viscosity of MR fluid also increase. Figure 1(d) shows that the MR fluid recovers rapidly and response time is only few milliseconds when the applied magnetic field disappeared.

Figure 2 shows the experimental device for recording holograms of MR fluid. The experimental conditions are as follows: the pixel number $N=1024$; the pixel size of the charge coupled device (CCD) camera $\Delta x=5.2 \mu \mathrm{m}$; the wavelength $\lambda=632.8 \mathrm{~nm}$; the magnification of objective $M=40$.

The hologram and reconstruction images of calibration target are shown in Figure 3. From Figure 3(b), we can easily obtain the length between two graduation lines based on counting the pixel numbers. Because the actual scale of calibration target is $50 \mu \mathrm{m}, M^{\prime}$ and actual $d$ can be calculated [13].

After a strong magnetic field is applied to MR fluids, the microparticles will be polarized and aligned like chains along the direction of magnetic field. The continuous holograms of MR fluids under an applied magnetic field were encoded by CCD and the construction images of the chain configuration are shown in Figure 4. The figures also indicate the transform process from micro-particles to chains in a magnetic field.
The chaining process along the direction of magnetic field, that is, the responding speed of MR fluids for magnetic field, is easily calculated.

2.2. Shear Yield Stress. In order to analyze the relationship between the shear yield stress and the magnetic field, the size, the volume percentage of MR fluid. The assumptions for chain model of dipole are as follows.

(1) The ordered arrangement of particles after magnetic polarization and the chain structure is steady. All of the particles occupy a fixed position in the stable chain.

(2) The single chain formed by particles is along with the direction of magnetic field. The chain is parallel to magnetic field direction, and its length is equal to the distance between two plates. All of the chains are the same in geometry, so the analysis results of arbitrary chain can be representative of the others.

(3) The acting force between adjacent particles in the chains is equal, which presents the tensile strength of chains.

(4) The adjacent particles are magnetized and turn into dipoles. The direction of the centerline of particles is parallel to the magnetic field.

(5) The interaction force in particles decides the strength of chains. When applied force is greater than the interaction force between particles, the chain will be pulled off. When the shear stress is perpendicular to the direction of magnetic field, the chain will be elongated and snapped.

(6) The particles are supposed to be spherosome and uniform.

The analysis mode of shear yield stress in MR fluid is shown in Figure 5, where $h$ represents the distance between two parallel plates and $F_{a}$ is the external force [14]. The bottom plate is fixed, and external force is applied to upper plate. When the shear stress is perpendicular to magnetic field direction, the chain will deform and break. The $\tau_{y}(H)$ represents the shear yield stress under unit area. The relationship between $\tau_{y}(H)$ and $F_{a}$ is indicated by $\tau_{y}(H)=F_{a} \sin \theta$, where $\theta$ represents the angle between the centerline of chain and magnetic field direction, as shown in Figure 5. 


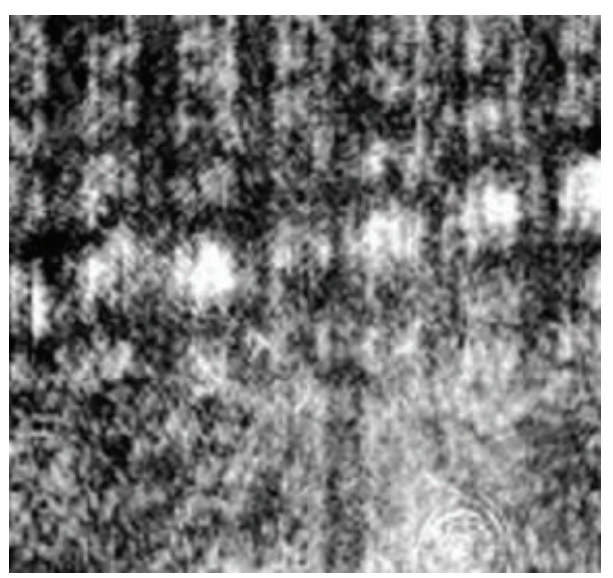

(a) Hologram

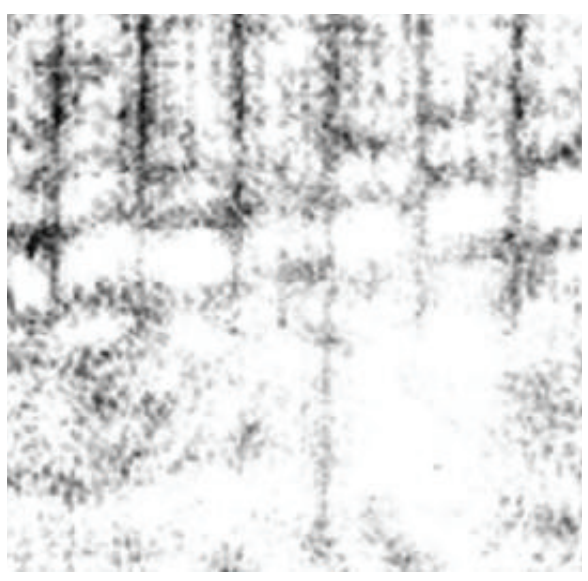

(b) Reconstruction image

FIGURE 3: Hologram and reconstruction images of calibration target.

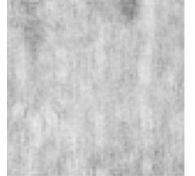

$1 s t$

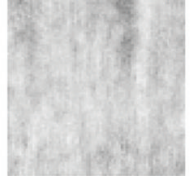

2nd

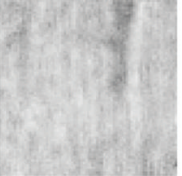

3 rd

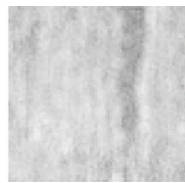

4 th

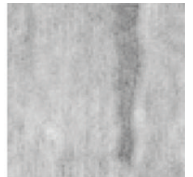

5 th

FIGURE 4: Reconstruction images (MR fluids under a magnetic field in different times).

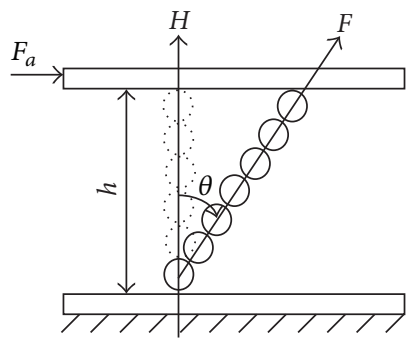

FIGURE 5: The analysis mode of shear yield stress.

With the applied magnetic field, the single magnetic particle is magnetized and forms dipoles in the MR fluid. The $J$ represents the dipole moment which can be expressed as follows [15]:

$$
J=\mu_{0} V_{1} M
$$

where $\mu_{0}$ is the permeability of vacuum, $V_{1}$ is the average volume of magnetic particles, $V_{1}=4 \pi r^{3} / 3$, and $M$ is magnetization intensity:

$$
M=\chi H
$$

where $\chi$ is the magnetic susceptibility and $H$ is the magnetic field strength.

The magnetic pole strength of the dipole can be expressed as follows:

$$
m=\frac{J}{2 r}
$$

The distance of dipoles which is formed by any two magnetic particles in the same chain is

$$
d=\frac{n(2 r+\delta)}{\cos \theta}
$$

where $\delta$ is the average value of the gap between two adjacent particles in the chain.

The average value of acting force in particles in the same chain can be expressed as follows:

$$
F=\frac{1}{4 \pi \mu_{0}} \frac{m^{2}}{d^{2}} .
$$

The shear yield stress of MR fluid under magnetic field is

$$
\tau_{y}(H)=\frac{N F \sin \theta}{A},
$$

where $A$ represents the area of the flat plate.

The number of chains in the unit area can be expressed as follows:

$$
N=\frac{\left(\phi A h / V_{1}\right)}{(h / R)},
$$

where $\phi$ is the volume fraction of magnetic particles in MR fluid and $R=2 r+\delta$.

Combining (1), (3), (4), and (6), the shear yield stress of MR fluid under magnetic fieldis is expressed as follows:

$$
\tau_{y}(H)=\sum_{n=1}^{k} \frac{\mu_{0}}{12 n^{2}} \frac{r \phi\left(\mu_{r}-1\right)^{2} H^{2}}{(2 r+\delta)} \sin \theta \cos ^{2} \theta,
$$




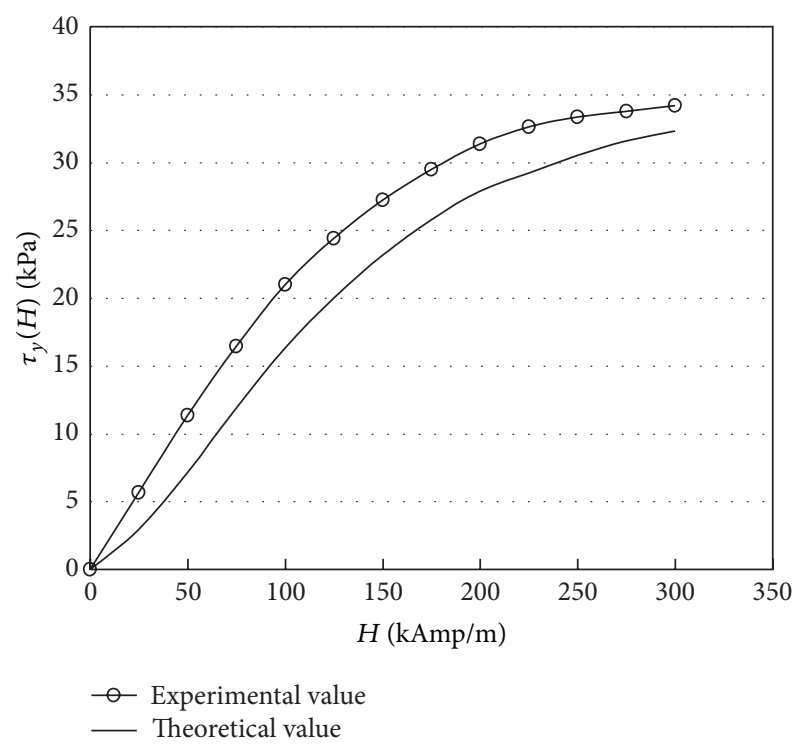

FIGURE 6: The yield stress versus applied magnetic field strength.

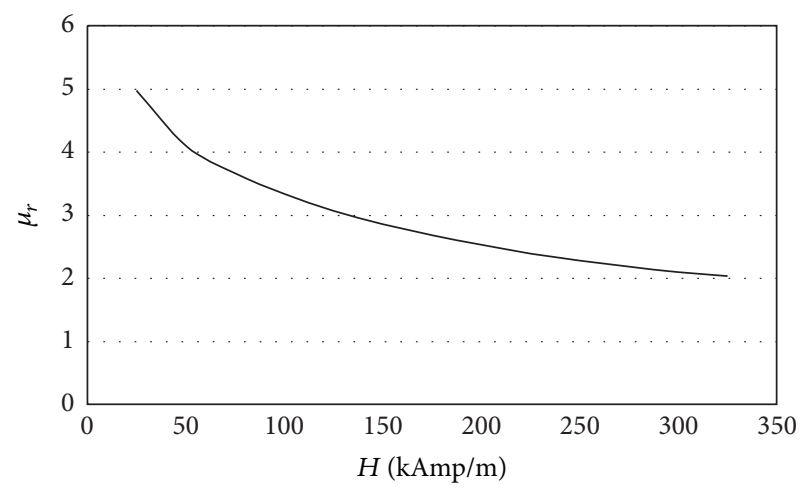

FIGURE 7: The relative magnetic permeability versus applied magnetic field strength.

where $\mu_{r}$ represents the relative magnetic permeability of MR fluid, $\mu_{r}=1+\chi, k$ represents the average number of particles in each chain, and $k=A h / V_{1} N$.

The theoretical value and experimental value of yield stress versus applied magnetic field strength are shown in Figure 6. The magnetic particle is uniform spherosome in the MR fluid. Assume that $\theta=30^{\circ}, \delta=0, \mu_{0}=4 \pi \times 10^{-7} \mathrm{Tm} / \mathrm{A}$, and $\phi=37 \%$. The relationship between the relative magnetic permeability and the applied magnetic field strength can be drawn, as shown in Figure 7. As shown in Figure 6, the theoretical value is satisfied with the experimental value, the yield stress of MR fluid is increased with the applied magnetic field and its value can be controlled by applied magnetic field.

\section{Rheological Properties of MR Fluid}

3.1. Test Equipment. The performance experimental device for rheological properties of MR fluid between two discs is shown in Figure 8. Based on this test system, the transmission torques of MR fluids between two discs under zero magnetic field and different applied magnetic fields are analyzed. The shearing rate of MR fluids between two discs can be adjusted by motor in the test system. The applied magnetic field strength can be controlled by electric current in coil. All parameters in system are measured in real time by gaussmeter, speed, and torque sensors.

3.2. Test Principle. For the properties of experimental system of MR fluid between two parallel disks, shown in Figure 8, the following assumptions are given: the fluid is incompressible. There is no flow in radial direction and axial direction, but only tangential flow. The flow velocity of MR fluid is a function of radius. The pressure in the thickness direction of MR fluid is constant. The strength of magnetic field in the gap of the activation region is well distributed. In cylindrical coordinates $(r, \theta, z)$, the distribution of the flow velocity is

$$
V_{r}=0, \quad V_{\theta}=r \omega(z), \quad V_{z}=0,
$$

where $V_{r}, V_{\theta}$, and $V_{z}$ are the flow velocity of the fluid in the $r$ direction, the $\theta$-direction, and $z$-direction, respectively; $\omega(z)$ is the rotation angular velocity of the fluid in the $\theta$-direction. The angular velocity $\omega(z)$ is the function of $z$-coordinate.

The fluid shear strain rate may be approximated as follows:

$$
\dot{\gamma}=\omega_{0} \frac{r}{h},
$$

where $\omega_{0}$ is speed of rotating disk. The torque transmitted by the MR fluid between two parallel disks is calculated by integrating the shear stress of the MR fluid as follows:

$$
T=2 \pi \int_{R_{1}}^{R_{2}} \tau r^{2} \mathrm{~d} r
$$

where $R_{1}$ and $R_{2}$ are the effective inner and outer radius of the rotor-disc in the MR fluid exposed to the magnetic field, respectively. Based on the mean value theorem of integral, the torque $T$ in (11) can be expressed as follows:

$$
T=\frac{2 \pi}{3} \tau^{*}\left(R_{2}^{3}-R_{1}^{3}\right)
$$

where $\tau^{*}$ is the shear stress of the sample. When $\left(R_{2}-R_{1}\right) \ll$ $R_{0}$, the $\tau^{*}$ is equal to the stress located at $R_{0}=\left(R_{2}-R_{1}\right) / 2$, approximately. Using (10) and (12), the relationship between $\tau$ and $\dot{\gamma}$ can be obtained by the measuring torque $T$ and speed $\omega_{0}$.

3.3. Test Results. In this experiment, we choose MR fluid samples MRM1, MRM2, and MRM3 to check the theory, as shown in Table 1 . Then we make a comparison with the results.

When the magnetic displacement is small, shown in Figure 9, magnetic particle is far from reaching a magnetic saturation and the shear stress quickly increases. With the increase of the magnetic induction intensity, curves gradually become slow. This is mainly because of different magnetisability of the solid ferromagnetism particles. We 


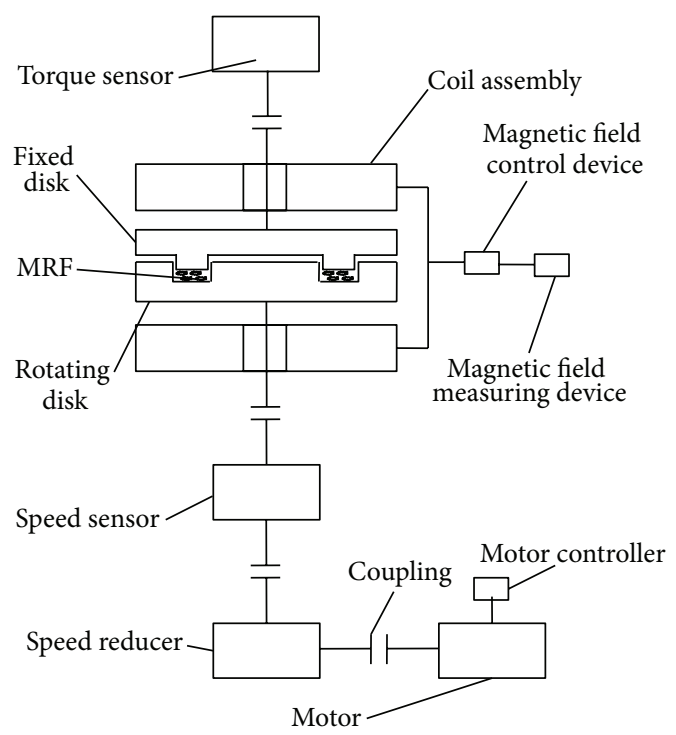

(a)

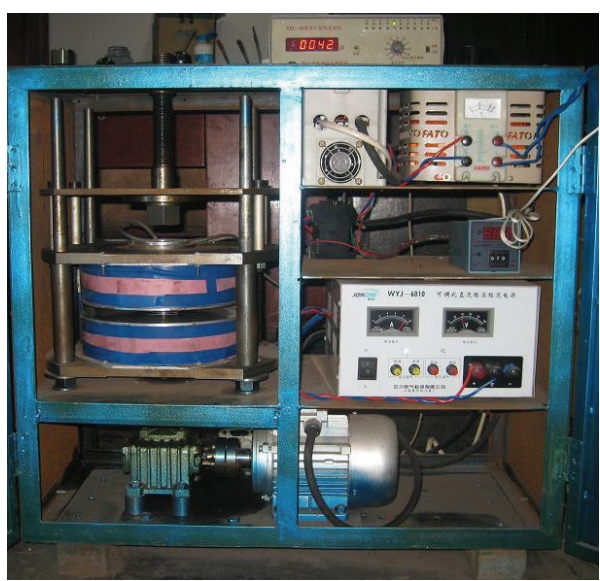

(b)

FIGURE 8: The performance experimental device for MR fluid rheological properties.

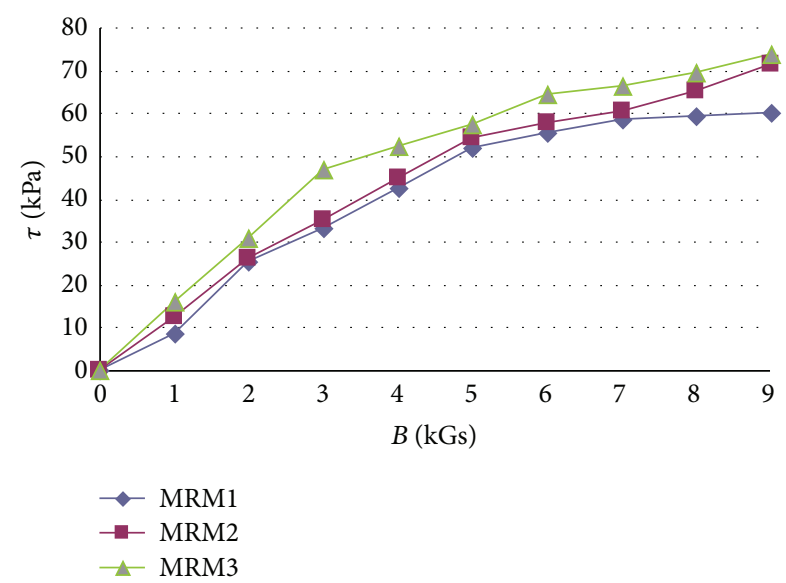

Figure 9: The shear stress of the different magnetic induction intensities.

TABLE 1: The MR fluid samples on different particle volume fractions.

\begin{tabular}{lcc}
\hline List & $\begin{array}{c}\text { Particle volume } \\
\text { fraction/\% }\end{array}$ & $\begin{array}{c}\text { Zero field } \\
\text { viscosity/(Pa.s) }\end{array}$ \\
\hline MRM1 & 5 & 0.1 \\
MRM2 & 15 & 0.2 \\
MRM3 & 35 & 1.1 \\
\hline
\end{tabular}

must increase the applied magnetic field strength in order to obtain a greater shear stress. If the magnetic induction intensity is large enough, the particles gradually reach magnetic saturation, particle interaction reaches the extreme value, and the shear stress at this time will not increase with the magnetic induction intensity and tends to a stable value.

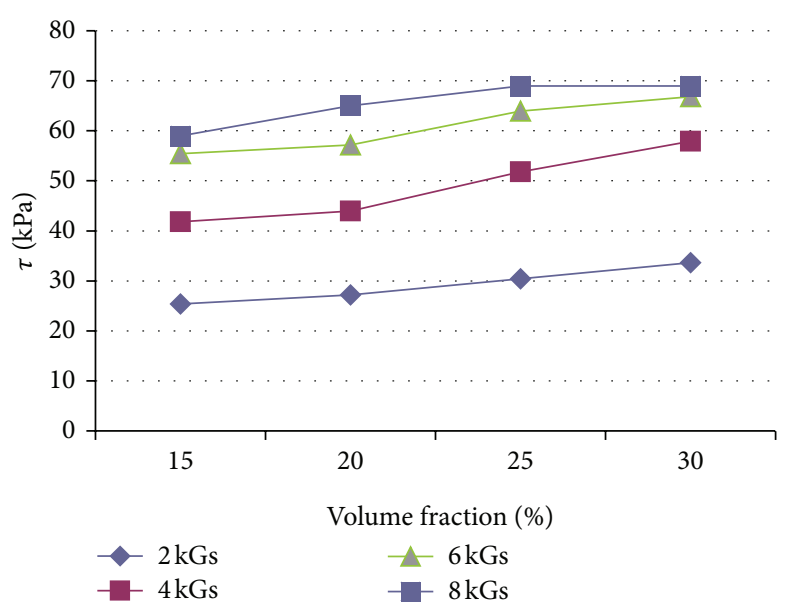

FIGURE 10: The shear stress of the different particle volume fractions.

The particles volume percentage refers to the percentage of the volume occupied by the dispersed phase of solid particles in the MR fluid. As Figure 10 shows, the shear stress also increases when the particle volume fraction increases. In the case of not very high magnetic field strength, both are rendering the approximate linear relationship. This can be explained by MR fluid microscopic mechanism. The solid particulate magnetic becomes dipole under the action of the magnetic field. Dipole of interaction form magnetic chain between the two plates. When the volume percentage is low, the number of solid particle is limited. In a magnetic field, a few of magnetic chains are formed and the shear stress is small. When the volume percentage is high, the number of magnetic chain increases and even forms column or mesh structure and the shear stress ensues to increase. 


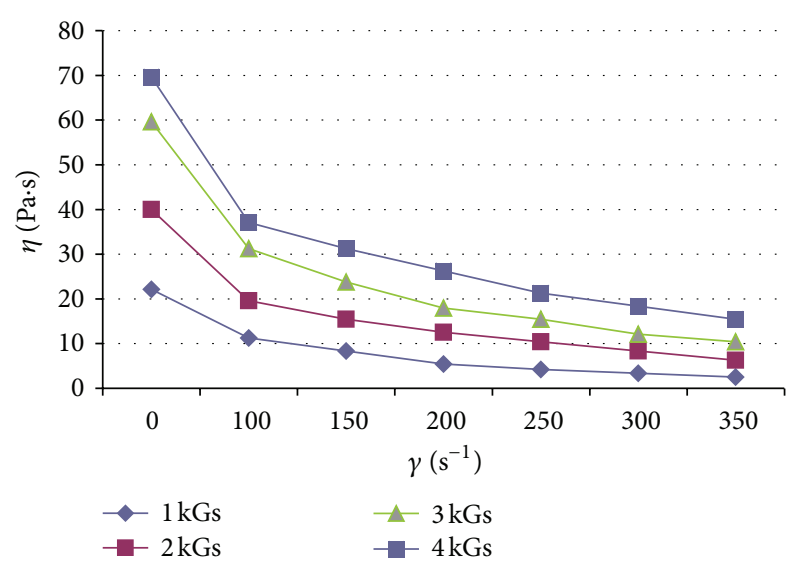

FIGURE 11: The relationship between viscosity and shear stress rate.

The apparent viscosity of MR fluid is the measure shear stress $\tau$ under certain conditions divided by the shear strain rate $\dot{\gamma}$. Obviously, for a Newtonian fluid, the apparent viscosity is the dynamic viscosity, and the value of viscosity is a constant, independent of the shear strain rate. But for MR fluid, it is not so. As Figure 11 shows, the apparent viscosity of MR fluid changes with shear strain rate under different magnetic induction intensity. The apparent viscosity decreases with increasing shear strain rate and in the beginning it decreased rapidly and then leveled off.

\section{Conclusion}

In order to predict the mechanical property of MR fluid under magnetic field and shear strain, the microstructures of chain at different magnetic fields strength were measured. The chain model of dipole interaction for MR fluid was established. The prediction model of yield stress for MR fluid is obtained. The influence of yield stress by magnetization intensity of magnetic particle and magnetic field strength were analyzed, respectively. In this experiment, we obtain the relationship between the shear stress and magnetic induction and particle volume fraction.

\section{Acknowledgments}

This work was financially supported by the National Natural Science Foundation of China (Grant no. 51175532); the Natural Science Foundation Key Project of Chongqing (Grant no. CSTC, 2011ba4028); Key Program of the Fundamental Research Funds for the Central Universities (Grant no. CDJXS10242206).

\section{References}

[1] G. L. Gulley and R. Tao, "Structures of a magnetorheological fluid," International Journal of Modern Physics B, vol. 15, no. 6-7, pp. 851-858, 2001.

[2] K. H. Song, B. J. Park, and H. J. Choi, "Effect of magnetic nanoparticle additive on characteristics of magnetorheological fluid," IEEE Transactions on Magnetics, vol. 45, no. 10, pp. 40454048, 2009.

[3] J. Huang, P. Wang, and G. Wang, "Squeezing force of the magnetorheological fluid isolating damper for centrifugal fan in nuclear power plant," Science and Technology of Nuclear Installations, vol. 2012, Article ID 175703, 6 pages, 2012.

[4] E. Dragašius, V. Grigas, D. Mažeika, and A. Šulginas, "Evaluation of the resistance force of magnetorheological fluid damper," Journal of Vibroengineering, vol. 14, no. 1, pp. 1-6, 2012.

[5] J. Huang, J. M. He, and J. Q. Zhang, "Viscoplastic flow of the MR fluid in a cylindrical valve," Key Engineering Materials, vol. 274-276, no. 1, pp. 969-974, 2004.

[6] A. M. Afonso, M. A. Alves, and F. T. Pinho, "Analytical solution of mixed electro-osmotic/pressure driven flows of viscoelastic fluids in microchannels," Journal of Non-Newtonian Fluid Mechanics, vol. 159, no. 1-3, pp. 50-63, 2009.

[7] P. Kielan, P. Kowol, and Z. Pilch, "Conception of the electronic controlled magnetorheological clutch," Przeglad Elektrotechniczny, vol. 87, no. 3, pp. 93-95, 2011.

[8] J. Huang, J. Q. Zhang, Y. Yang, and Y. Q. Wei, "Analysis and design of a cylindrical magneto-rheological fluid brake," Journal of Materials Processing Technology, vol. 129, no. 1-3, pp. 559-562, 2002.

[9] J. Noma, H. Abe, T. Kikuchi, J. Furusho, and M. Naito, "Magnetorheology of colloidal dispersion containing Fe nanoparticles synthesized by the arc-plasma method," Journal of Magnetism and Magnetic Materials, vol. 322, no. 13, pp. 1868-1871, 2010.

[10] C. Ekwebelam and H. See, "Microstructural investigations of the yielding behaviour of bidisperse magnetorheological fluids," Rheologica Acta, vol. 48, no. 1, pp. 19-32, 2009.

[11] K. I. Jang, J. Seok, B. K. Min, and S. J. Lee, "Behavioral model for magnetorheological fluid under a magnetic field using Lekner summation method," Journal of Magnetism and Magnetic Materials, vol. 321, no. 9, pp. 1167-1176, 2009.

[12] J. Pacull, S. Gonçalves, Á. V. Delgado, J. D. G. Durán, and M. L. Jiménez, "Effect of polar interactions on the magnetorheology of silica-coated magnetite suspensions in oil media," Journal of Colloid and Interface Science, vol. 337, no. 1, pp. 254-259, 2009.

[13] F. F. Fang, H. J. Choi, and M. S. Jhon, "Magnetorheology of soft magnetic carbonyl iron suspension with single-walled carbon nanotube additive and its yield stress scaling function," Colloids and Surfaces A, vol. 351, no. 1-3, pp. 46-51, 2009.

[14] S. Chen, K. Jian, and X. Peng, "Cylindrical magnetorheological fluid variable transmission controlled by shape-memory alloy," Science and Technology of Nuclear Installations, vol. 2012, Article ID 856082, 6 pages, 2012.

[15] X. Z. Zhang, X. L. Gong, P. Q. Zhang, and Q. M. Wang, "Study on the mechanism of the squeeze-strengthen effect in magnetorheological fluids," Journal of Applied Physics, vol. 96, no. 4, pp. 2359-2364, 2004. 

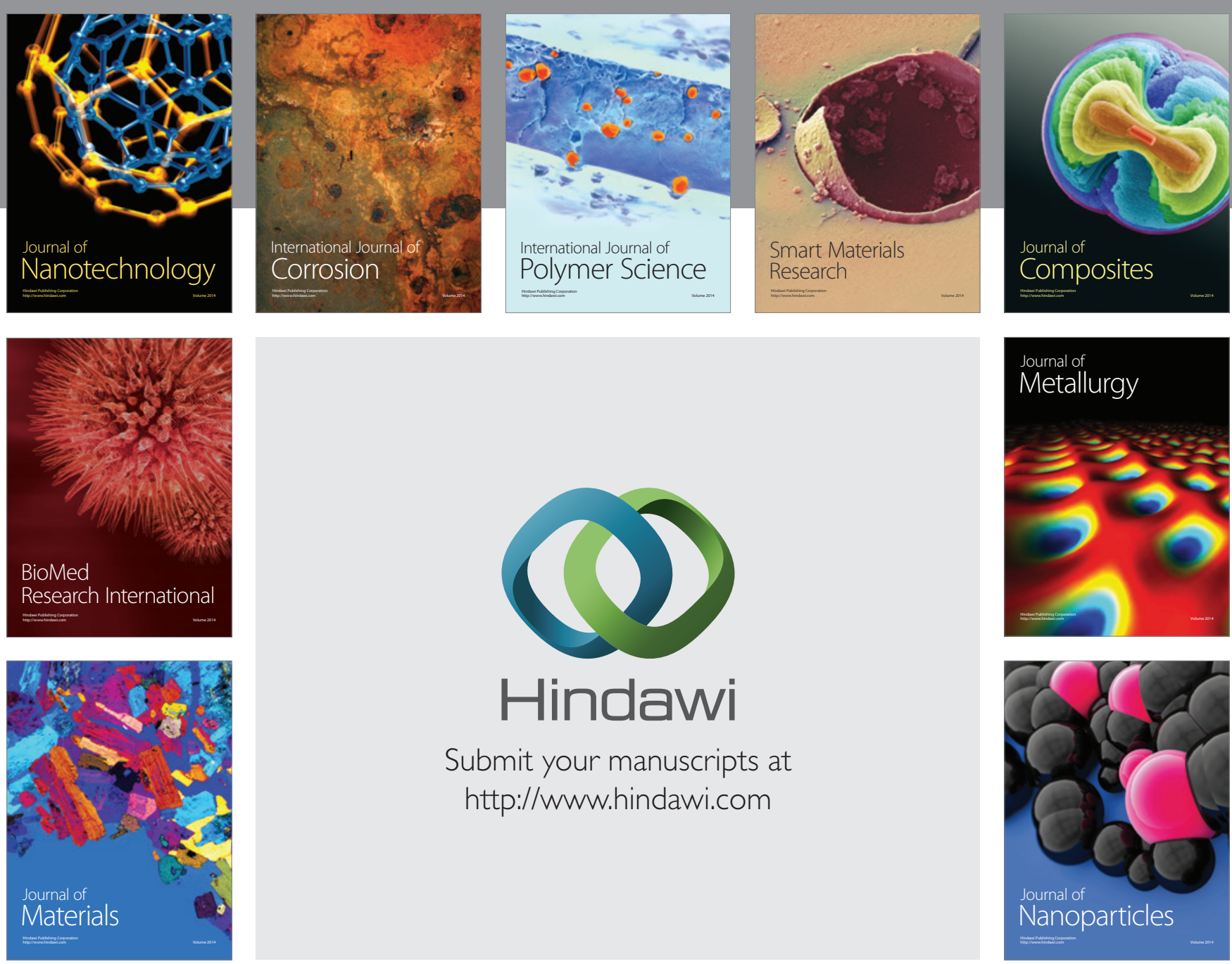

Submit your manuscripts at http://www.hindawi.com
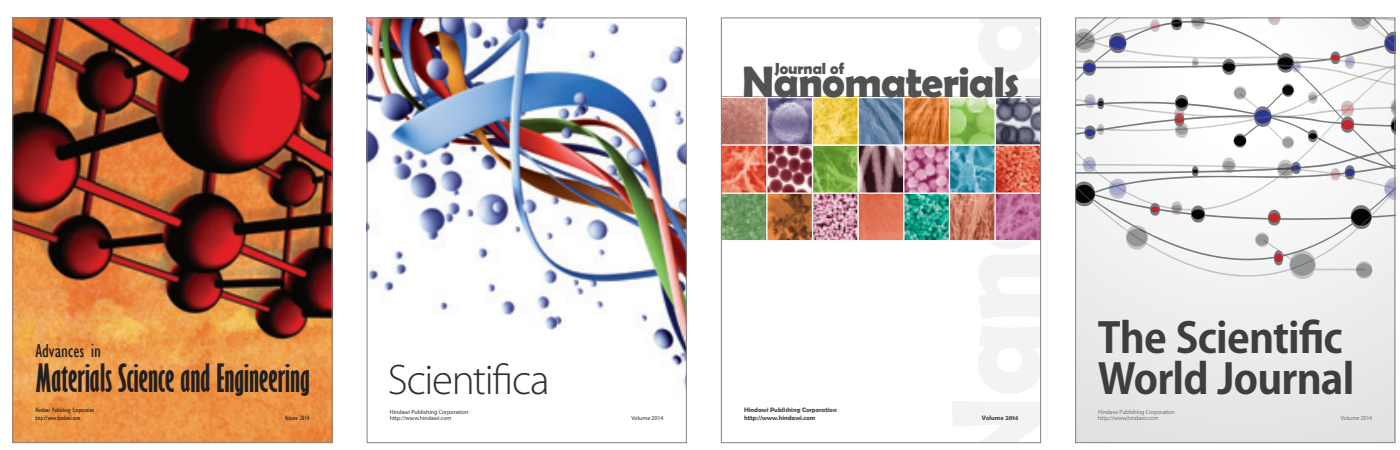

\section{The Scientific World Journal}
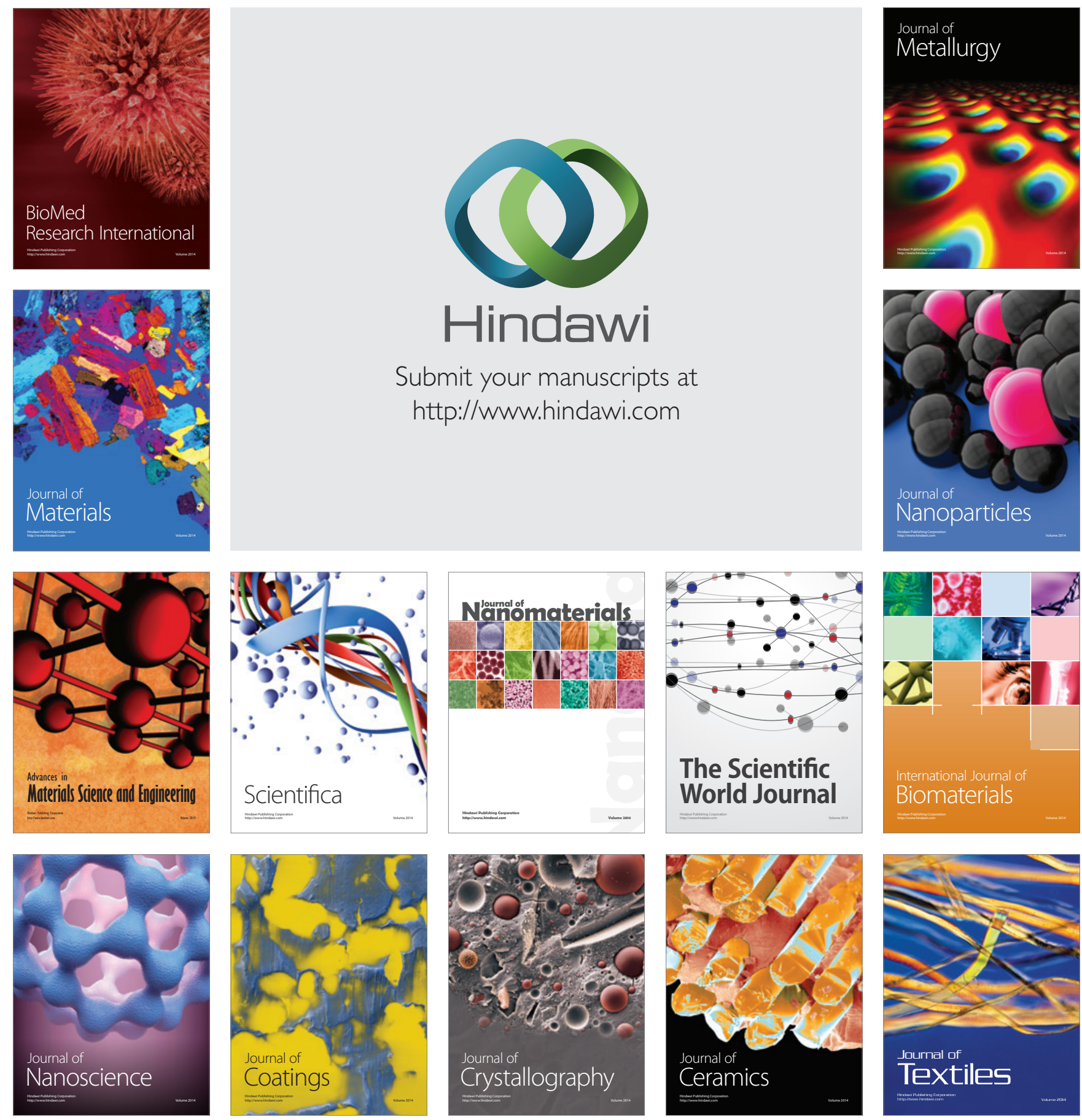